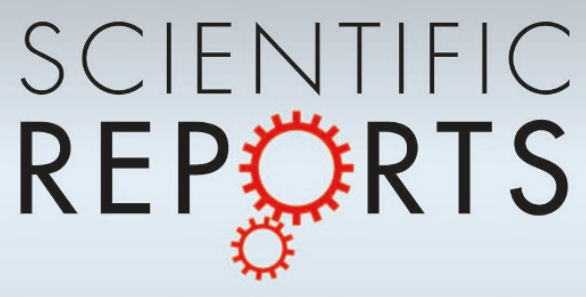

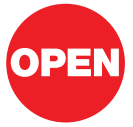

SUBJECT AREAS: CONDENSED MATTER PHYSICS

OPTICAL MATERIALS AND STRUCTURES

PHOTOCHEMISTRY

SPECTROSCOPY

Received

4 May 2011

Accepted

19 August 2011

Published

8 September 2011

Correspondence and requests for materials should be addressed to A.D.M. (amohite@lanl.

gov)

\section{Quasi-Molecular Fluorescence from Graphene Oxide}

\author{
Charudatta Galande', Aditya D. Mohite ${ }^{2}$, Anton V. Naumov',4, Wei Gao', Lijie Ci' , Anakha Ajayan', \\ Hui Gao', Anchal Srivastava ${ }^{5}$, R. Bruce Weisman ${ }^{4}$ \& Pulickel M. Ajayan ${ }^{1,4}$
}

\begin{abstract}
'Department of Mechanical Engineering and Materials Science, Rice University, Houston, TX 77005, USA, ${ }^{2}$ Center for Integrated Nanotechnologies and Chemistry Division, Los Alamos National Laboratory, Los Alamos, NM 87545, USA, ${ }^{3}$ Applied Physics Program, Rice University, Houston, TX, USA, ${ }^{4}$ Department of Chemistry, Rice University, Houston, TX, USA, ${ }^{5}$ Department of Physics, Banaras Hindu University, Varanasi, India.
\end{abstract}

Aqueous dispersions of graphene oxide (GO) have been found to emit a structured, strongly $\mathrm{pH}$-dependent visible fluorescence. Based on experimental results and model computations, this is proposed to arise from quasi-molecular fluorophores, similar to polycyclic aromatic compounds, formed by the electronic coupling of carboxylic acid groups with nearby carbon atoms of graphene. Sharp and structured emission and excitation features resembling the spectra of molecular fluorophores are present near $500 \mathrm{~nm}$ in basic conditions. The GO emission reversibly broadens and red-shifts to ca. $680 \mathrm{~nm}$ in acidic conditions, while the excitation spectra remain very similar in shape and position, consistent with excited state protonation of the emitting species in acidic media. The sharp and structured emission and excitation features suggest that the effective fluorophore size in the GO samples is remarkably well defined.

S ince its discovery in $2004^{1}$, graphene has been the focus of intense fundamental and applied research. Its unique $2 \mathrm{D}$ dispersion leads to superior properties such as ballistic transport at room temperature, high carrier mobility, and broad-band optical transitions, which could be exploited in a wide range of applications ${ }^{2}$. However, graphene is a zero-gap semiconductor and controllably modifying the electronic band structure remains a major challenge. Thus, most optoelectronic applications of graphene have been limited to its integration as a passive element (e.g. transparent conductive electrodes in solar cells and light emitting diodes) ${ }^{3}$. Two methods for modifying the band structure of graphene have been pursued. One is cutting graphene into nanoribbons $^{4,5,6}$ and carbon quantum dots ${ }^{7,8}$. The optical properties of these structures are not well understood. The second is chemical functionalization, which offers a variety of approaches for controllably modulating the graphene band structure. One such functionalized product is graphene oxide (GO). As reported by several groups, GO shows significant optical absorption and broadband fluorescence in the visible and near-infrared (NIR) spectral regions ${ }^{9,10,11}$. As a result, GO has received attention for its potential use in low cost, large area optoelectronic applications. In contrast to pristine graphene, in which all atoms are $\mathrm{sp}^{2}$ hybridized, GO also contains $\mathrm{sp}^{3}$ carbon atoms covalently bonded to oxygen-bearing functional groups ${ }^{12,13,14}$. Theoretical studies on model systems predict an optical band gap and fluorescence due to localization in the $\pi$-system ${ }^{15,16,17}$. The broadband fluorescence observed in GO has been attributed to $\pi$-electrons confined in localized sp ${ }^{2}$ domains $s^{9,10,11}$. Chhowalla and co-workers ${ }^{11}$ reported that moderately reduced GO films excited in the ultraviolet emit broad blue emission attributed to such localized domains. These previous reports consider functionalization only as a source of electronic disorder in graphene. However, Gokus et al. ${ }^{18}$ have recently observed visible luminescence from oxygen plasma-treated graphene and attributed the emission to $\mathrm{CO}$-related localized graphene electronic states. Here we report molecular-like fluorescence from GO that is spectrally structured and strongly $\mathrm{pH}$-dependent. These findings support the view that covalently bound oxygen species interact with surrounding carbon atoms of the graphene sheet to create localized, quasi-molecular electronic structures in GO.

\section{Results}

Our as-prepared graphene oxide suspension was mildly acidic ( $\mathrm{pH}$ 5.2) due to traces of acid remaining from the synthesis (see Methods). Emission measured with $440 \mathrm{~nm}$ excitation at this $\mathrm{pH}$ showed a broad peak (FWHM > 200nm) centred near $668 \mathrm{~nm}$ (Fig. 1a), similar to spectra previously reported ${ }^{9,10,11,18}$. As $\mathrm{pH}$ was increased from highly acidic ( $\mathrm{pH}$ 1.7) toward neutral values, we observed a monotonic decrease in the intensity of this emission peak, with no significant change in shape (Fig. 1a). Increasing $\mathrm{pH}$ in the basic range above $\mathrm{pH} 7$ led to the 

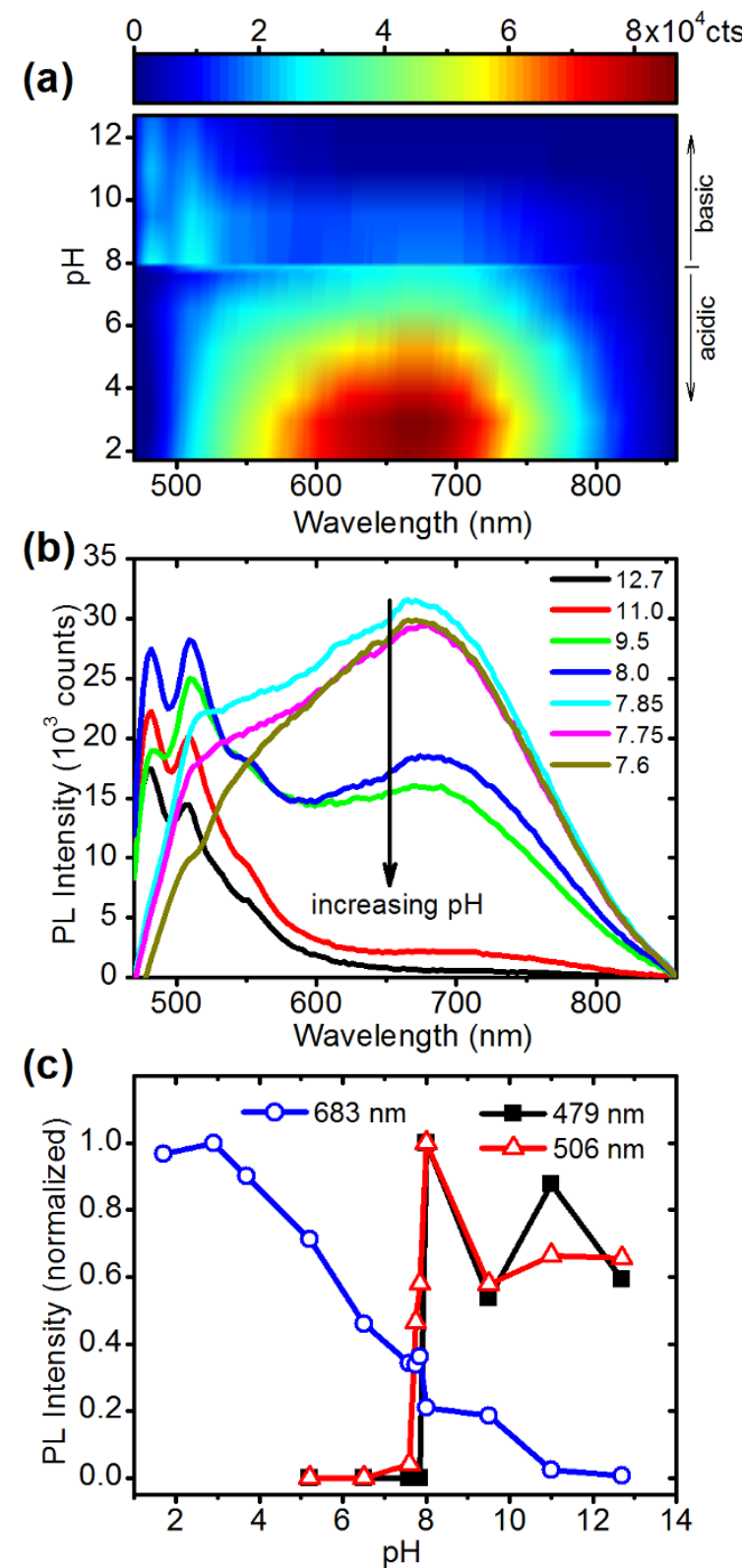

Figure $1 \mid \mathrm{pH}$ dependent fluorescence spectra of graphene oxide. (a) Emission spectra measured with $440 \mathrm{~nm}$ excitation for sample $\mathrm{pH}$ values between 1.7 and 12.7. (b) Traces from a measured in the basic range from pH 7.6 to 12.7, plotted on an expanded scale for clarity. (c) Intensities of emission components peaking at 479,506, and $683 \mathrm{~nm}$ (deduced from spectral simulations) as a function of sample $\mathrm{pH}$.

disappearance of the $668 \mathrm{~nm}$ feature and the appearance of two relatively sharp peaks near $482 \mathrm{~nm}(\sim 16 \mathrm{~nm}$ FWHM $)$ and $506 \mathrm{~nm}$ ( $\sim 26 \mathrm{~nm}$ FWHM) (Fig. 1b). By simulating the set of emission spectra as superposition of five Gaussian components, we deduced corrected peak wavelengths of 479,506,531, 577, and $683 \mathrm{~nm}$ (with significant uncertainties in the peak positions of the broad 577 and $683 \mathrm{~nm}$ features). (Fig. S-3) A plot of deduced amplitudes of the three major peaks $(479,506$, and $683 \mathrm{~nm})$ vs. $\mathrm{pH}$ shows that the acidic $(683 \mathrm{~nm})$ peak decreases in intensity smoothly and almost monotonically from $\mathrm{pH} 1.7$ to 12.7 , while the two basic peaks (479 and $506 \mathrm{~nm}$ ) emerge abruptly in the narrow $\mathrm{pH}$ range between 7.6 to 8.0 , as in a fluorimetric titration (Fig. 1c). The observed changes in fluorescence spectra were completely reversible with $\mathrm{pH}$ within the measured range, indicating no loss of oxygen species. ${ }^{19}$
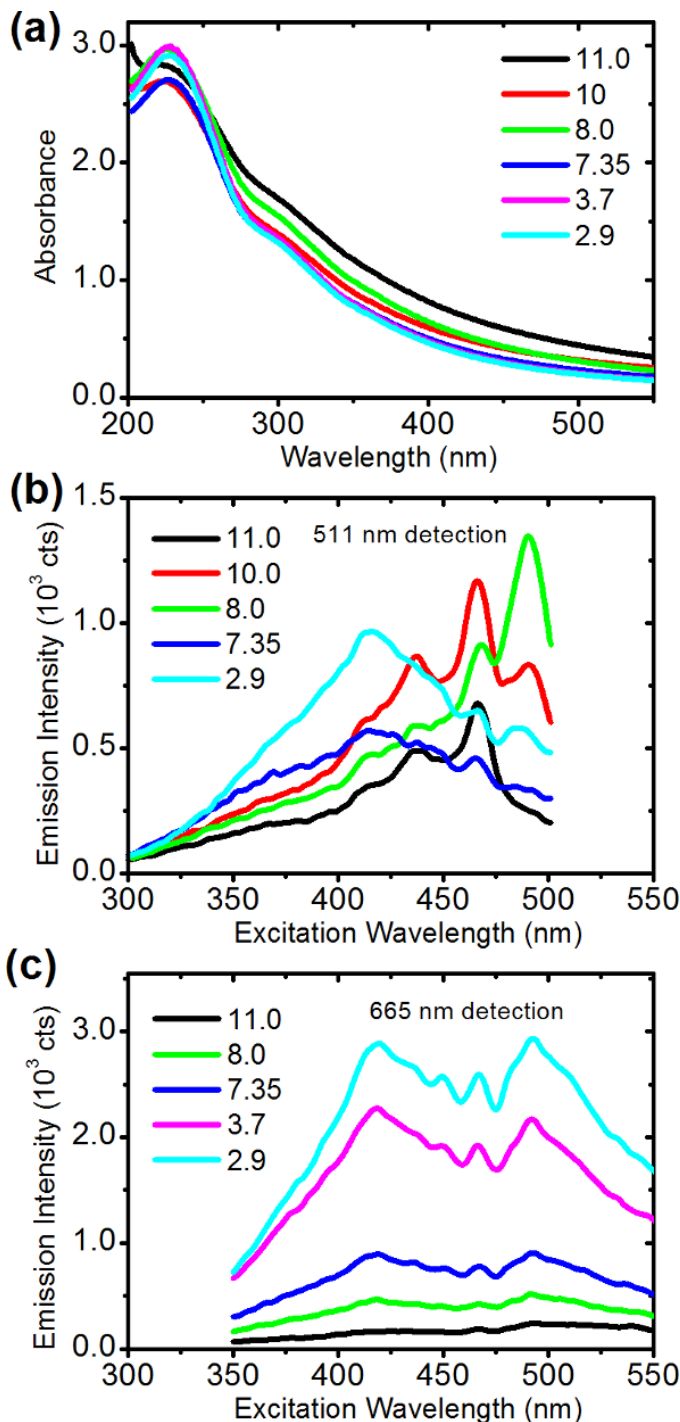

Figure $2 \mid$ Graphene oxide absorbance and excitation spectra. (a) Absorbance spectra ( $1 \mathrm{~cm}$ path length) measured at $\mathrm{pH}$ values between 2.9 and 11. (b) Excitation spectra measured for emission at $511 \mathrm{~nm}$, for sample $\mathrm{pH}$ values between 2.9 and 11. (c) Excitation spectra measured for emission at $665 \mathrm{~nm}$, for sample $\mathrm{pH}$ values between 2.9 and 11 .

We investigated further using absorption and fluorescence excitation spectroscopies. Absorption traces between 200 and $550 \mathrm{~nm}$ for GO suspensions at $\mathrm{pH} 2.9$ to $\mathrm{pH} 11$ showed no clear features except for a peak near $230 \mathrm{~nm}$ (Fig. 2a). This is likely from $\pi$-plasmon absorption, although its $5.4 \mathrm{eV}$ energy is somewhat higher than observed for SWCNTs or graphene. ${ }^{20}$ The absorption tail at longer wavelengths was significantly more intense at higher $\mathrm{pH}$ values. We found no evidence of sample aggregation or instability during these measurements.

Measured $\mathrm{pH}$ variations in the GO absorption spectra were minor compared to those found in emission, suggesting that the dominant absorbing species in the sample are not the fluorophores. This is confirmed by the fluorescence excitation spectra (Fig. 2b,c), which differ greatly from the absorption spectra. Under basic conditions, the emission feature at $506 \mathrm{~nm}$ was induced by rather distinct excitation bands near 491, 465, 436, and $417 \mathrm{~nm}$. Similar behaviour was found for $479 \mathrm{~nm}$ emission (Fig. S-4). As pH increased above 8, there was a striking reduction in the efficiency with which the $491 \mathrm{~nm}$ excitation induced the $506 \mathrm{~nm}$ emission. Fluorescence excitation spectra of the broad (acidic) emission feature near $683 \mathrm{~nm}$ showed distinct peaks matching the positions of those found in excitation 
spectra of the basic emission (Fig. 2c). The fact that the different GO emission features seen under acidic and basic conditions are excited by matching transitions indicates that both types of emission arise from the excitation of the same ground state species. In a separate experiment, the photocurrent action spectrum of GO measured using an innovative capacitive technique ${ }^{21}$ showed photocurrent peaks corresponding to the excitation peaks in Fig. $2 \mathrm{~b}$ and $2 \mathrm{c}$.

\section{Discussion}

Analysis of the data in Figs. 1 and 2 for GO samples under basic $\mathrm{pH}$ conditions revealed molecular-like patterns in the structured emission and excitation spectra. However, these patterns were more complex than are seen from pure molecular samples. For $\mathrm{GO}$ at $\mathrm{pH} 8$, we observed that $511 \mathrm{~nm}$ emission was efficiently induced by $491 \mathrm{~nm}$ excitation, but when the sample was adjusted to $\mathrm{pH} 11$ and excited at a shorter wavelength, it emitted at $479 \mathrm{~nm}$. The presence of emission at a shorter wavelength than an excitation peak indicates that the GO sample contained more than one fluorophore. By plotting normalized excitation and emission spectra separately at the two $\mathrm{pH}$ values, we found two pairs of near-mirror-image spectra, labeled A and B (Fig. 3a). Each of these excitation/emission pairs resembles data from a molecular fluorophore. In this interpretation, the electronic origin
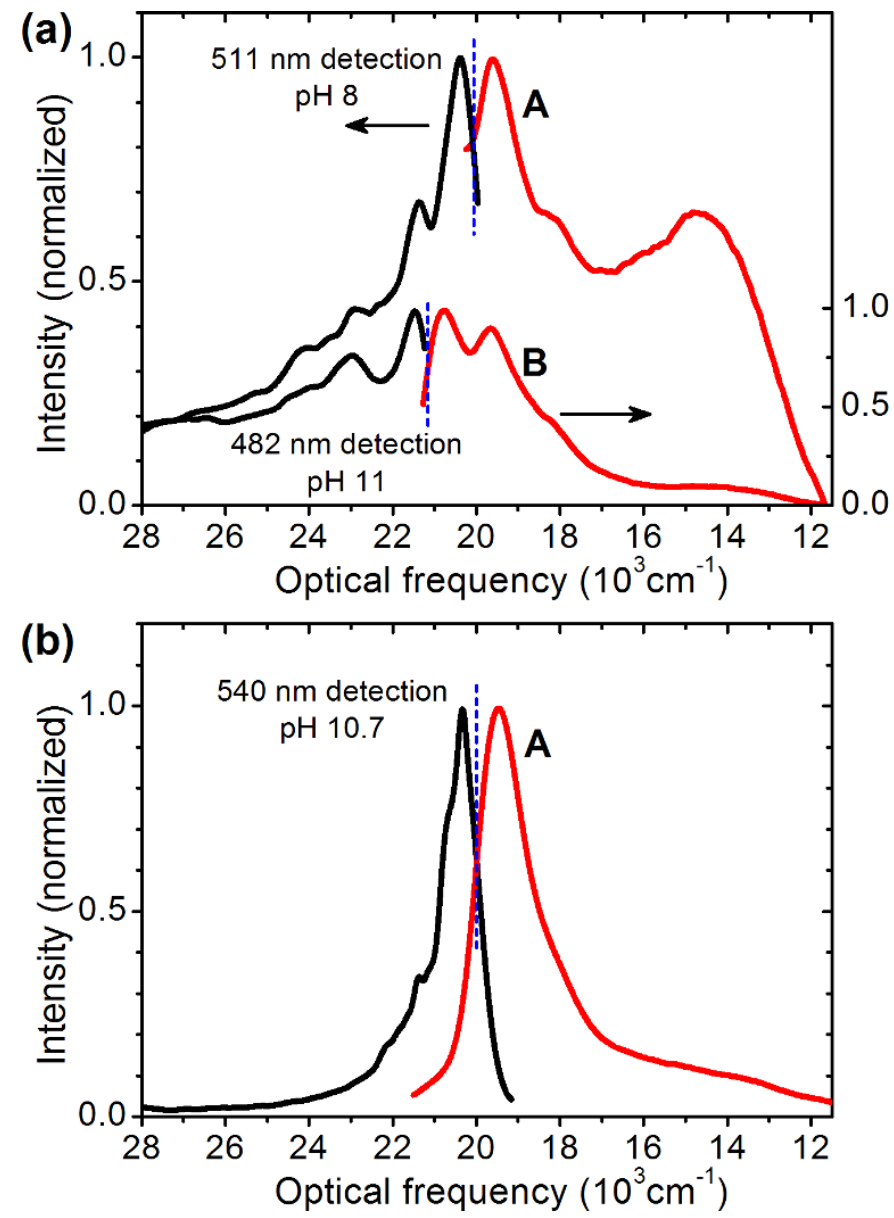

Figure 3 Combined excitation and emission spectra of graphene oxide. (a) Normalized fluorescence and excitation spectra for GO samples at pH 8 (traces labeled A) and pH 11 (traces labeled B). Fluorescence spectra were recorded with $440 \mathrm{~nm}$ excitation for both $\mathrm{A}$ and $\mathrm{B}$; excitation spectra were measured with $511 \mathrm{~nm}$ detection for $A$, and $482 \mathrm{~nm}$ detection for $B$. (b) Normalized fluorescence and excitation spectra for a suspension of GO prepared from expandable graphite. The sample $\mathrm{pH}$ was 10.7. The fluorescence spectrum was measured with $440 \mathrm{~nm}$ excitation, and the excitation spectrum was measured with $540 \mathrm{~nm}$ detection. frequencies of species A and B would be 20,050 and $21190 \mathrm{~cm}^{-1}$, respectively, and their fluorescence Stokes shifts (the spacing between main emission and excitation peaks) would be approximately $650 \mathrm{~cm}^{-1}$. Although the chemical composition of GO varies from batch to batch, we found that all samples show similar fluorescence-excitation patterns in basic media. The data also contain secondary spectral features separated by $\sim 1000$ to $1600 \mathrm{~cm}^{-1}$, which are suggestive of short vibrational progressions in molecular electronic spectra.

The view that the spectra in Fig. 3a represent more than one fluorophore is supported by comparable measurements on a GO sample prepared from intercalated graphite, a slightly different starting material (Fig. 3b, Fig. S-5). Spectra of this sample show peaks that match the main features of traces A in Fig. 3a but no peaks corresponding to $\mathrm{B}$. We therefore deduce that $\mathrm{A}$ and $\mathrm{B}$ represent distinct species that have different relative abundances in the two samples. Further, the absence of pronounced side bands in Fig. $3 \mathrm{~b}$ suggests that the side bands in Fig. 3a may arise from additional fluorophores in the sample rather than vibronic transitions of species A. A more detailed analysis of these additional fluorophores is beyond the scope of this work as the transitions at $531 \mathrm{~nm}$ and $577 \mathrm{~nm}$ (Fig. S-3) are not distinct and are apparent only on spectral fitting.

Vibrational absorption spectra were measured using ATR-FTIR spectroscopy of solid GO samples obtained from acidic and basic suspensions (Fig. 4). A number of absorption bands are evident. These are assigned to vibrational modes in groups including $\mathrm{COOH}, \mathrm{COO}^{-}, \mathrm{C}-\mathrm{OH}, \mathrm{C}-\mathrm{H}$, and $\mathrm{C}=\mathrm{O}$ (see Fig. 4 caption). ${ }^{22}$ Among these, we would particularly expect $\mathrm{COOH}$ and $\mathrm{COO}^{-}$ groups to exhibit $\mathrm{pH}$-dependent behaviour over the studied sample conditions.

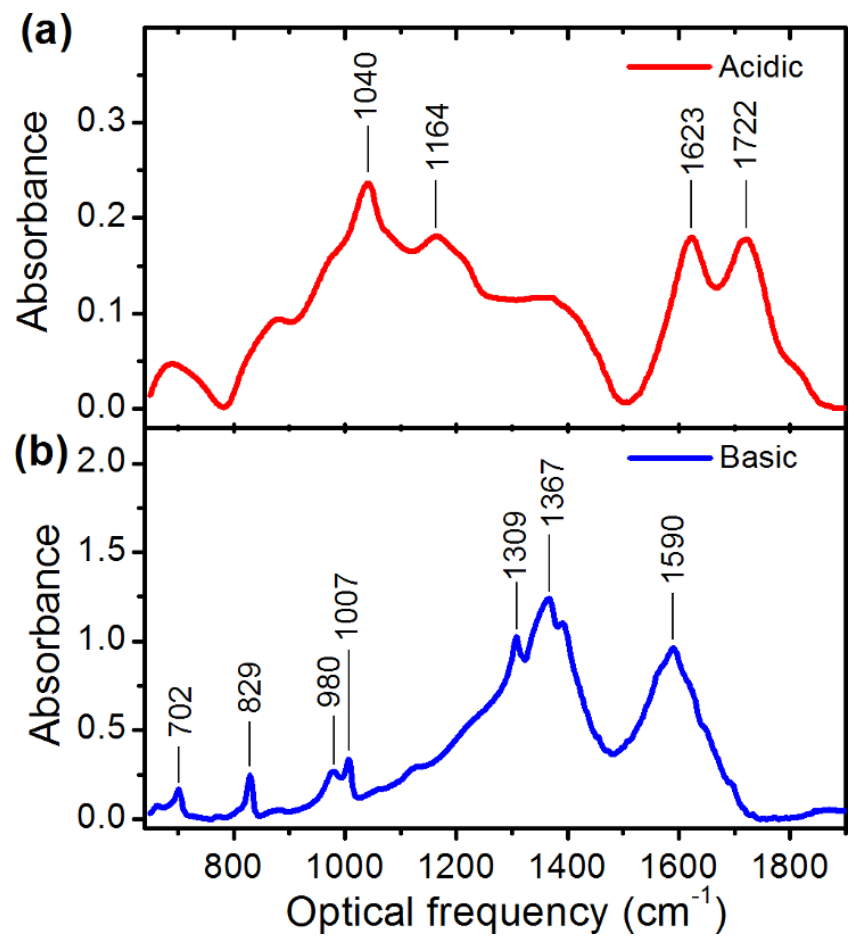

Figure $4 \mid$ Vibrational absorption spectra of graphene oxide. ATR-FTIR spectra of solid GO samples isolated from (a) acidic and (b) basic aqueous suspensions. Major bands and proposed assignments in (a): $1040 \mathrm{~cm}^{-1}$ (C-O stretch) , $1164 \mathrm{~cm}^{-1}$ (C-OH stretch), $1623 \mathrm{~cm}^{-1}$ (adsorbed water \& skeletal vibrations of unoxidized graphitic domains), $1722 \mathrm{~cm}^{-1}(\mathrm{C}=\mathrm{O}$ stretch); in (b): $829 \mathrm{~cm}^{-1}$ (C-H out-of-plane wag), $980 \mathrm{~cm}^{-1}$ (possibly epoxide stretch), $1007 \mathrm{~cm}^{-1}$ (C-H in-plane bend), $1309 \mathrm{~cm}^{-1}$ (C-O stretch), $1367 \mathrm{~cm}^{-1}\left(\mathrm{COO}^{-}\right.$symmetric stretch), $1590 \mathrm{~cm}^{-1}$ (COO antisymmetric stretch). 
Although GO is a complex non-stoichiometric material that incorporates multiple oxygen-bearing addends, it may be useful to interpret its $\mathrm{pH}$-dependent fluorescence spectroscopy by analogy to polycyclic aromatic carboxylic acids. Model compounds would then include pyrene-3-carboxylic acid (3-PCA), pyrene-1-carboxylic acid (1-PCA), or anthracic acids (9-AC, 1-AC, 2-AC). Our observation that the same excitation transitions cause very different emission spectra in basic and acidic media indicates the presence of excited state proton transfer. The tendencies for ground and excited state deprotonation are described by values of $\mathrm{pK}_{\mathrm{a}}$ and $\mathrm{pK}_{\mathrm{a}}{ }^{*}$, respectively. For the model compounds listed above, it is known that $\mathrm{pK}_{\mathrm{a}}{ }^{*}$ is higher than $\mathrm{pK}_{\mathrm{a}}$ by approximately 1.5 to 5 units (3.8 vs. 5.2 for $3-\mathrm{PCA}^{23}, 4.0$ vs. 8.7 for $1-\mathrm{PCA}^{24,25}, 3.0$ vs. 6.5 for $\left.9-\mathrm{AC}^{26,27}\right)$. Thus, at $\mathrm{pH}$ values between $\mathrm{pK}_{\mathrm{a}}$ and $\mathrm{pK}_{\mathrm{a}}{ }^{*}$, carboxylic acid groups would be largely deprotonated to ionic $-\mathrm{COO}^{-}$groups in the ground state but reprotonated in the electronically excited state. We suggest that the structured emission seen above $\mathrm{pH} 8$ in our GO samples arises from electronically excited $\left(\mathrm{G}-\mathrm{COO}^{-}\right) *$ moieties formed by excitation of ground state $\mathrm{G}-\mathrm{COO}^{-}$. The abrupt loss of short-wavelength emission bands below $\mathrm{pH} 8$ (Fig. 1c) would then be associated with excited state protonation of the $\left(\mathrm{G}-\mathrm{COO}^{-}\right)^{*}$ to $(\mathrm{G}-\mathrm{COOH})^{*}$, which contribute to the broad, red-shifted emission seen under acidic conditions. We note that the observed $\mathrm{pH}$-dependence and spectral structure of GO fluorescence are qualitatively very similar to those reported for several aromatic carboxylic acids such as $3-\mathrm{PCA}^{23}, 1-\mathrm{PCA}^{24,25}, 9-\mathrm{AC}$, 1 -AC, and $2-\mathrm{AC}^{26,27,28}$ in polar solvents, where structured mirrorimage excitation/emission spectra were observed at basic $\mathrm{pH}$ but only broad red-shifted fluorescence at acidic $\mathrm{pH}$. The reversibility of fluorescence spectral changes with $\mathrm{pH}$ in our GO samples rules out aggregation as a cause of the red-shifted acidic emission. The broad, strongly red-shifted emission of carboxylic acids in the acidic medium has been attributed ${ }^{23,24,27,28}$ to a combination of strong interaction of the protonated acid with the polar solvent and large geometric changes in the protonated species during excitation and emission.

GO is a heterogeneous material. Apart from the presence of addends other than carboxylic acid groups, there must also be a distribution of distances between addend positions and a variety of sites (e.g. face or edge) at which they are located. In view of this complexity, it is remarkable that the fluorescence spectroscopy of GO appears as simple as it does. We suggest that the GO fluorescence is dominated by a small number of quasi-molecular fluorophores that may consist of isolated $\mathrm{COOH}$ groups and the set of nearby graphene atoms that they significantly perturb. To investigate this possibility, we have performed semi-empirical (PM3) quantum calculations on graphene sheet fragments containing 240 carbon atoms and one $\mathrm{COOH}$ or $\mathrm{COO}^{-}$group bonded to an edge atom. Our results for $\mathrm{COO}^{-}$show a delocalized but relatively distinct region of negative electrostatic potential (Fig. 5), which we interpret as the region of strong electronic coupling between the graphene sheet and addend group. Although the range of coupling is not sharply defined, it seems largely confined to a section containing approximately 34 carbon atoms (13 hexagonal rings). The computed electronic absorption spectra of the $\mathrm{COOH}$ and $\mathrm{COO}^{-}$graphene adducts show several intense singlet-singlet transitions between 450 and $550 \mathrm{~nm}$ and fewer transitions between 650 and $750 \mathrm{~nm}$ (Fig. S-6). These wavelengths are quite similar to those found experimentally. We note that if the GO fluorophore is considered analogous to a polycyclic aromatic hydrocarbon, the observed sharp transitions would correspond to a molecule containing approximately $50 \pi$-electrons, which is reasonably consistent with the perturbed graphene region estimated from our computational modelling. More extensive calculations will be valuable for clarifying the nature of optical transitions involving coupled graphene $\pi$-electrons and oxygen-containing addends such as carboxylic acids.

In summary, we have found strongly $\mathrm{pH}$-dependent visible fluorescence from aqueous dispersions of graphene oxide. In basic

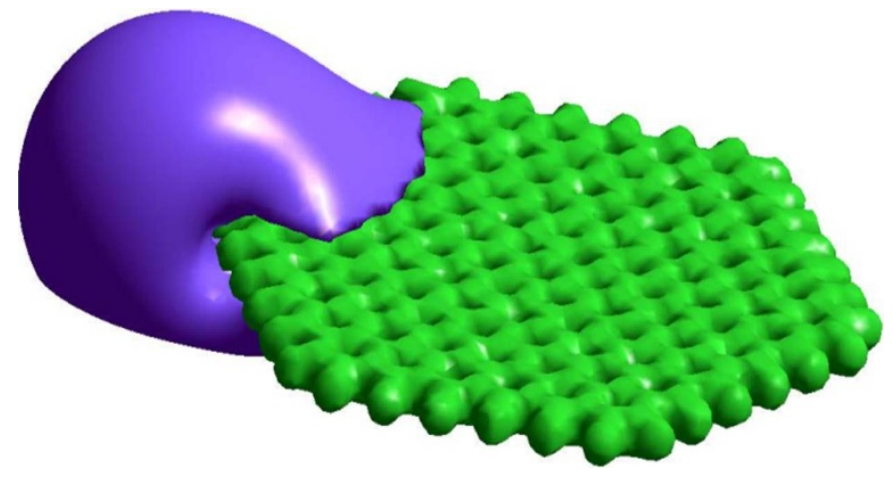

Figure $5 \mid$ Computed electrostatic potential isosurface for a graphene sheet fragment derivatized at its edge with $\mathrm{COO}^{-}$. The semiempirical quantum chemical calculation was performed using the PM3 method with a graphene fragment containing 240 atoms. The plotted purple isosurface, enclosing the $\mathrm{COO}^{-}$group, shows the boundary of the region in which there is a negative potential value exceeding $0.06 \mathrm{e} / \mathrm{a}_{0}$.

conditions, relatively sharp and structured emission and excitation features appear near $500 \mathrm{~nm}$. These resemble spectra from a mixture of polycyclic aromatic compounds. In acidic conditions, the ca. $500 \mathrm{~nm}$ fluorescence is replaced by broad and strongly red-shifted emission peaking near $680 \mathrm{~nm}$, while the excitation spectra show little change in shape. Our findings point to excited state protonation of the fluorophore species in acidic media. Assuming that the oxygen bearing addends are sufficiently isolated and that there are no major energy transfer effects, we propose, based on experimental results and model computations, that the observed spectral features arise from carboxylic acid groups electronically coupled with nearby atoms of the graphene sheet to form quasi-molecular fluorophores similar to polycyclic aromatic compounds. Spectra suggest that the effective fluorophore size in the GO samples is remarkably well defined. It may prove possible to further enhance the homogeneity of such local electronic modifications of graphene through refined chemical processing methods. Further experimental investigations, including time-resolved spectroscopy should be useful to elucidate the nature of GO fluorescence.

\section{Methods}

The starting material was commercially available graphite powder (SP-1 graphite, from Bay Carbon Corporation). GO was obtained by oxidation of the graphite powder by the modified Hummers method ${ }^{29,30}$. In a separate batch, Expandable Graphite (Intumescent Flake Graphite, from Asbury Carbons) was first thermally exfoliated at $700^{\circ} \mathrm{C}$ for $1 \mathrm{~min}$ and was further used to synthesize GO by the modified Hummer's method. GO was suspended in deionised water $(0.1 \mathrm{mg} / \mathrm{mL})$ by bath ultrasonication for $30 \mathrm{~min}$ followed by tip sonication for $10 \mathrm{~min}$ at $5 \mathrm{~W}$ to form a clear yellow suspension. No particles were visible after sonication and the suspension remained stable for several weeks, indicating that the GO flakes were small and well dispersed. The suspension was then divided into $10 \mathrm{~mL}$ aliquots and their $\mathrm{pH}$ values were adjusted between 1.7 and 12.7 by addition of dilute $\mathrm{H}_{2} \mathrm{SO}_{4}$ or dilute $\mathrm{KOH}$. Just before each measurement, the sample was tip sonicated at $5 \mathrm{~W}$ for $5 \mathrm{~min}$.

Fluorescence measurements were performed on a J-Y Spex Fluorolog 3-211 spectrofluorometer using $7 \mathrm{~nm}$ spectral slit widths for excitation and emission, $3 \mathrm{~nm}$ step sizes, and integration times of $6 \mathrm{~s}$ per point. Emission spectra were recorded using $440 \mathrm{~nm}$ excitation. Measured spectra were corrected for detector response and excitation power. A spectrum of DI water was used as a blank to correct for solvent Raman scattering. Absorbance was measured in quartz cuvettes on a Cary 4000 spectrophotometer. To prepare samples for FTIR spectroscopy, the pH 1.7 suspension was further acidified until the GO started to aggregate (at $\mathrm{pH} \sim 0.5$ ), and then centrifuged at $13,000 \mathrm{x}$ g for $20 \mathrm{~min}$ to obtain a solid pellet of acidic GO. A pellet of basic GO (from $\mathrm{pH} \sim 13.5$ ) was obtained in a similar manner. FTIR spectra were measured on a Nicolet FTIR Microscope in the ATR mode with an MCT/A detector. TEM images and selected area electron diffraction patterns (Fig. S1) were obtained with a JEOL 2100 Field Emission Gun TEM.

1. Novoselov, K. S. et al. Electric Field Effect in Atomically Thin Carbon Films. Science 306, 666-669 (2004). 
2. Geim, A. K. \& Novoselov, K. S. The rise of graphene. Nature Mater 6, 183-191 (2007).

3. Bonaccorso, F., Sun, Z., Hasan, T. \& Ferrari, A. C. Graphene photonics and optoelectronics. Nature Photon 4, 611-622 (2010).

4. Han, M. Y., Brant, J. C. \& Kim, P. Electron Transport in Disordered Graphene Nanoribbons. Phys. Rev. Lett. 104, 056801 (2010).

5. Wang, X. et al. Room-Temperature All-Semiconducting Sub-10-nm Graphene Nanoribbon Field-Effect Transistors. Phys. Rev. Lett. 100, 206803 (2008).

6. Jiao, L., Zhang, L., Wang, X., Diankov, G. \& Dai, H. Narrow graphene nanoribbons from carbon nanotubes. Nature 458, 877-880 (2009).

7. Schnez, S. et al. Observation of excited states in a graphene quantum dot. Appl. Phys. Lett. 94, 012107 (2009).

8. Mueller, M. L., Yan, X., McGuire, J. A. \& Li, L. Triplet States and Electronic Relaxation in Photoexcited Graphene Quantum Dots. Nano Letters 10, 2679$2682(2010)$

9. Sun, X. et al. Nano-graphene oxide for cellular imaging and drug delivery. Nano Research 1, 203-212 (2008).

10. Luo, Z., Vora, P. M., Mele, E. J., Johnson, A. T. C. \& Kikkawa, J. M. Photoluminescence and band gap modulation in graphene oxide. Appl. Phys. Lett. 94, 111909 (2009)

11. Eda, G. et al. Blue Photoluminescence from Chemically Derived Graphene Oxide. Adv. Mater. 22, 505-509 (2010).

12. Gao, W., Alemany, L. B., Ci, L. \& Ajayan, P. M. New insights into the structure and reduction of graphite oxide. Nature Chem 1, 403-408 (2009).

13. Bagri, A. et al. Structural evolution during the reduction of chemically derived graphene oxide. Nature Chem 2, 581-587 (2010).

14. Dreyer, D. R. et alThe chemistry of graphene oxide Chem. Soc. Rev. 39, 228-240 (2010)

15. Mathioudakis, C. et al. Electronic and optical properties of a-C from tight-binding molecular dynamics simulations. Thin Solid Films 482, 151-155 (2005).

16. Chen, C. W. \& Robertson, J. Nature of disorder and localization in amorphous carbon. J. Non-Cryst. Solids 227-230, 602-606 (1998).

17. Robertson, J. \& O’Reilly, E. P. Electronic and atomic structure of amorphous carbon. Phys. Rev. B 35, 2946 (1987).

18. Gokus, T. et al. Making Graphene Luminescent by Oxygen Plasma Treatment. ACS Nano 3, 3963-3968 (2009).

19. Fan, X., Peng, W., Li, Y., Li, X., Wang, S., Zhang, G. and Zhang, F. Deoxygenation of Exfoliated Graphite Oxide under Alkaline Conditions: A Green Route to Graphene Preparation. Adv. Mater. 20, 4490-4493 (2008).

20. Rance, G. A., Marsh, D. H., Nicholas, R. J. \& Khlobystov, A. N. UV-vis absorption spectroscopy of carbon nanotubes: Relationship between the [pi]-electron plasmon and nanotube diameter. Chem. Phys. Lett. 493, 19-23 (2010).

21. Mohite, A. D., Santos, T. S., Moodera, J. S. \& Alphenaar, B. W. Observation of the triplet exciton in EuS-coated single-walled nanotubes. Nat Nano 4, 425-429 (2009)

22. Socrates, G. Infrared and Raman Characteristic Group Frequencies: Tables and Charts. (John Wiley and Sons, 2004).

23. Milosavljevic, B. H. \& Thomas, J. K. Solvent effects on the photophysical properties of pyrene-3-carboxylic acid. J. Phys. Chem. 92, 2997-3001 (1988).

24. Nucci, N. V., Zelent, B. \& Vanderkooi, J. M. Pyrene-1-Carboxylate in Water and Glycerol Solutions: Origin of the Change of pK Upon Excitation. J Fluoresc 18, 4149 (2007).
25. Zelent, B., Vanderkooi, J. M., Coleman, R. G., Gryczynski, I. \& Gryczynski, Z. Protonation of Excited State Pyrene-1-Carboxylate by Phosphate and Organic Acids in Aqueous Solution Studied by Fluorescence Spectroscopy. Biophysical Journal 91, 3864-3871 (2006).

26. Donckt, E. V. \& Porter, G. Acidity constants of aromatic carboxylic acids in the S1 state. Trans. Faraday Soc. 64, 3215-3217 (1968).

27. Werner, T. C. \& Hercules, D. M. Fluorescence of 9-anthroic acid and its esters. Environmental effects on excited-state behavior. The Journal of Physical Chemistry 73, 2005-2011 (1969).

28. Werner, T. C. \& Hercules, D. M. Charge-transfer effects on the absorption and fluorescence spectra of anthroic acids. The Journal of Physical Chemistry 74, 1030-1037 (1970).

29. Hummers, W. S. \& Offeman, R. E. Preparation of Graphitic Oxide. Journal of the American Chemical Society 80, 1339 (1958).

30. Kovtyukhova, N. I. et al. Layer-by-Layer Assembly of Ultrathin Composite Films from Micron-Sized Graphite Oxide Sheets and Polycations. Chemistry of Materials 11, 771-778 (1999).

\section{Acknowledgements}

R.B.W. and A.V.N. thank the National Science Foundation (grant CHE-0809020) and the Welch Foundation (grant C-0807) for support. C.G., A.D.M., and P.M.A. acknowledge support from Rice University startup funds. P.M.A. and L.C. acknowledge funding support from the Office of Naval Research (ONR) through the MURI program on graphene (Award N00014- 09-1-1066). A.D.M. acknowledges the Center for Integrated Nanotechnologies, Los Alamos National Laboratory for support and Andrew Dattelbaum, Jason Han and Han Htoon for valuable discussions. A.S. acknowledges the support from Department of Science and Technology (DST), GOI, under BOYSCAST fellowship.

\section{Author Contributions}

A.D.M. conceived the experiment and co-wrote the manuscript. C.G. \& A.V.N. performed the experiments, data analysis, and co-wrote the manuscript. A.V.N. performed the semi-empirical calculations. C.G., W.G. and H.G. synthesized the different GO samples. L.C. performed TEM analysis. A.A. performed initial absorbance measurements. A.S. contributed to synthesis of intercalated GO samples. R.B.W. interpreted data and co-wrote the manuscript. P.M.A. discussed the results and commented on the manuscript. All authors contributed to the manuscript.

\section{Additional information}

Competing financial interests The authors declare no competing financial interests.

Supplementary information accompanies this paper at http://www.nature.com/ scientificreports

License: This work is licensed under a Creative Commons

Attribution-NonCommercial-NoDerivative Works 3.0 Unported License. To view a copy of this license, visit http://creativecommons.org/licenses/by-nc-nd/3.0/

How to cite this article: Galande, C. et al. Quasi-Molecular Fluorescence from Graphene Oxide. Sci. Rep. 1, 85; DOI:10.1038/srep00085 (2011) 